Muséologies

Les cahiers d'études supérieures

muséologies

\title{
Une mise en crise du musée, un entretien avec Christian Bernard, directeur du Musée d'art moderne et contemporain à Genève (Mamco)
}

\section{Anne Bénichou et Francine Couture}

Volume 5, numéro 1, automne 2010

URI : https://id.erudit.org/iderudit/1033523ar

DOI : https://doi.org/10.7202/1033523ar

Aller au sommaire du numéro

Éditeur(s)

Association Québécoise de Promotion des Recherches Étudiantes en Muséologie (AQPREM)

ISSN

1718-5181 (imprimé)

1929-7815 (numérique)

Découvrir la revue

Citer ce document

Bénichou, A. \& Couture, F. (2010). Une mise en crise du musée, un entretien avec Christian Bernard, directeur du Musée d'art moderne et contemporain à Genève (Mamco). Muséologies, 5(1), 86-105. https://doi.org/10.7202/1033523ar 
UN

Anne Bénichou et Francine Couture

Une mise en crise du musée, un entretien avec Christian Bernard, directeur du Musée d'art moderne et contemporain à Genève (Mamco)

JUILLET 2008, REVU EN SEPTEMBRE 2010

Christian Bernard, né en 1950 à Strasbourg, est le directeur du Mamco (Musée d'art moderne et contemporain de Cenève), musée d'un nouveau type qu'il a conçu et mis en oeuvre en 1994. C. Bernard a publié de nombreux articles, préfaces et entretiens, tant en France et en Suisse quailleurs en Europe. 11 a donné de nombreuses conférences et participé à de nombreux jurys, colloques, tables-rondes et séminaires en Suisse et en Europe. De 1986 à 1994, il a dirigé la Villa Arson, établissement du Ministère de la Culture français qui réunit un Centre national d'art contemporain, une école d'art expérimentale et des résidences d'artistes à Nice. II a été Conseiller pour les arts plastiques auprès de la Direction régionale des Affaires culturelles Rhône-Alpes à Lyon, de 1982 à 1985. A ce titre, il a contribué à créer la collection régionale d'art contemporain (FRAC), suscité d'importantes commandes publiques (Richard Serra à Bourg-en-Bresse, projet Jean-Pierre Raynaud aux Minguettes, etc.) et accompagné la mise en place de grandes infrastructures pour l'art contemporain. 


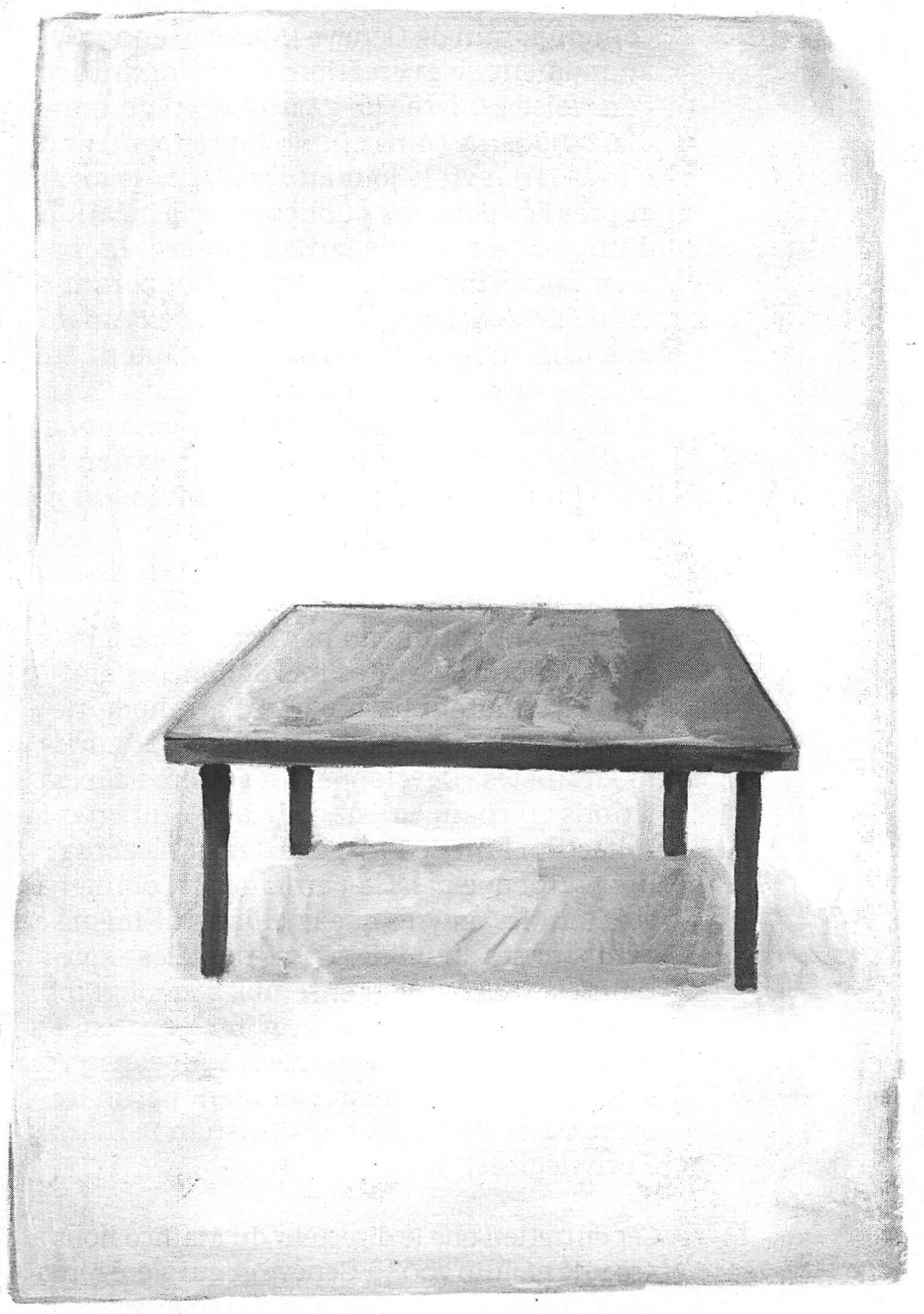


Après avoir tenu les rênes de la Villa Arson à Nice durant huit ans, Christian Bernard a pris la direction du Musée d'art moderne et contemporain de Genève (Mamco) en 1994, au moment de sa création. Cette institution genevoise est issue de l'Association du musée d'art moderne (Amam), un regroupement de citoyens qui vit le jour en 1973, afin de militer auprès des pouvoirs publics pour la création d'un musée d'art moderne à Cenève. En 1991, la municipalité aux prises avec d'importantes difficultés financières délégua à l'Amam une usine désaffectée, l'actuel bâtiment du musée, sans autres formes de soutien. C'est dans ce contexte de crise économique et de mobilisation citoyenne que l'association invita Christian Bernard à concevoir un projet de musée.

Il le pensa comme un espace de réflexion et d'expérimentation qui permettrait de dépasser les apories maintes fois dénoncées auxquelles se butent les musées d'art moderne et contemporain calqués sur les paradigmes modernistes. Développer un système d'expositions en changement continu, y intégrer la collection afin de l'inscrire dans une actualité plutôt que dans la permanence, donner à voir tant les œuvres que le musée lui-même en exhibant ses stratégies et ses modèles expositionnels, préférer des ensembles monographiques à l'échantillonnage, inviter les artistes à collaborer aux espaces qui leur sont consacrés et à leur développement comptent parmi les principales stratégies que Christian Bernard a privilégiées.

Cet entretien que le directeur du Mamco nous a accordé en juin 2008 à Genève et qui a été remanié à l'automne 2010 porte sur la collection du musée, ses orientations, et les modalités très 
inventives de ses développements et de sa mise en exposition. Il a été réalisé dans le cadre d'un projet de recherche sur les pratiques de réexposition et sur les variations des œuvres contemporaines. Le Mamco nous est apparu exemplaire de l'action réciproque des pratiques artistiques contemporaines sur la culture muséale. D'une part, l'institution a installé une collaboration avec les artistes en leur donnant la parole dans la conception et de leurs espaces monographiques en constante transformation ; d'autre part, les stratégies expositionnelles du Mamco ont contribué à l'analyse de l'exposition comme un médium et un intermédiaire qui exerce une action sur la production de l'œuvre d'art dans l'espace public. 


\section{Anne Bénichou et Francine Couture Quels sont les principes de travail ou les axes de réflexion qui sous-tendent le Mamco?}

\section{Christian Bernard}

Il faudrait développer de nombreux points. Certains tiennent aux conditions spécifiques de la création du Mamco. Il s'agissait au départ de concevoir et de mettre en œuvre un musée d'art contemporain sans disposer de collection préalablement constituée, dans un bâtiment industriel de type fonctionnaliste classique (quatre plateaux d'environ $1000 \mathrm{~m}^{2}$ superposés) qui ne présente pas que des avantages pour une activité expositionnelle, dans un contexte politique réticent et un cadre budgétaire extrêmement limité. Ce projet était porté depuis 1973 par une association, épaulée depuis 1991 par une fondation privée qui réunissait des citoyens (aisés) décidés à pallier les carences de la ville. Cette pauvreté initiale était aussi une chance: on m'a donné liberté entière de concevoir une institution qui n'était pas tributaire de l'attente d'un retour sur investissement, ni du politique ni du privé. Notre précarité, notre totale incertitude quant à notre avenir me sont apparues autant comme une condition que comme une visée: mettre en mouvement un musée de crise qui ferait de l'instabilité l'un de ses moyens majeurs. Musée de crise au sens économique, mais aussi musée de la crise du musée, de mise en crise du musée. Un des aspects de la crise du musée est l'épuisement de ses paradigmes modernes. D'où le projet de structurer le Mamco selon un spectre assez large de types de "lieux pour l'art": de l'étroit cabinet au vaste plateau en passant par le loft (nous avons par exemple une salle nommée Loft Don Judd), du dépôt à la crypte en passant par l'atelier, le studio ou l'appartement. Ces types, extérieurs au musée traditionnel, y introduisent une forme d'hétérogénéité critique. Un autre élément fondateur du Mamco a été l'accent mis sur les espaces monographiques permanents ou récurrents. Cela change radicalement le travail du musée car les ensembles monographiques impliquent des choix, des engagements durables qui s'opposent aux logiques d'échantillonnage ou de course aux artistes des canons marchand et historiographique actuels. Pour 
évoquer encore une orientation initiale du Mamco, j'insisterais sur la volonté de considérer le musée non comme un thésaurus, mais comme un processus continu de production d'une offre expositionnelle, en constant réexamen, en permanente reconfiguration. Si un lieu aussi singulier que la Crypte de Claudio Parmiggiani n'a aucune vocation à changer, si un dispositif comme la reconstitution de l'appartement de Chislain Mollet-Viéville a peu évolué (mais des données nouvelles y apparaissent discrètement trois fois par an), tous les autres espaces du musée n'ont cessé d'être modifiés dans leur présentation et même dans leur forme ou leur localisation. Le Mamco que l'on visite n'est jamais qu'un moment réversible de son "identité en devenir".

\section{Anne Bénichou et Francine Couture}

Il y a donc au Mamco de nombreux espaces monographiques, des salles dédiées à l'œuvre d'un seul artiste, qui présentent des corpus assez imposants de leur production, et dont les configurations évoluent régulièrement. D'où vient votre intêrêt pour les espaces monographiques?

\section{Christian Bernard}

L'insistance sur l'espace monographique permanent ou récurrent se comprend en regard d'une certaine et assez ancienne histoire des relations contrariées entre des artistes et le musée. Gustave Moreau est sans doute l'un des premiers qui en ait tiré la leçon dans un geste de retrait splendide. Plus près de nous, Don Judd a formulé des conclusions radicales tant dans ses écrits que dans ses actes (son immeuble de SoHo et la Fondation Chinati à Marfa) dont peu d'institutions se sont inspirés, sinon la Dia ou Insel Hombroich à Neuss. Voilà des références toujours nourricières pour moi. L'enjeu de l'insistance monographique est évidemment à la fois artistique, éthique et politique. On pourrait songer aussi aux retraites fleuries de Giverny ou de Seebüll (Monet et Nolde), ou encore à Michael Heizer et son entreprise démesurée dans son ranch du Nevada. Walter de Maria appartient à sa manière à cette lignée des excentrés. Rien ne nous est plus salubre aujourd'hui que ces exemples d'artistes produisant des lieux pour l'art (le leur ou celui de leurs affinités électives), ces auto-commanditaires 
indépendants du musée comme machine de monstration, de production et de conservation. Bien avant d'être en situation d'imaginer le Mamco, je visitais leurs sites pour échapper au tournant spectaculaire-marchand des musées d'art des années 1980-1990. C'est dans cet esprit que j'ai souhaité associer très tôt au projet du Mamco des artistes comme Franz Erhard Walther, Claudio Parmiggiani, Siah Armajani, Sarkis ou Claude Rutault, par exemple.

\section{Anne Bénichou et Francine Couture}

Certains espaces monographiques procèdent d'une délégation de programmation à l'artiste, établie selon des modalités très différentes selon les cas. Comment travaillez-vous avec les artistes?

\section{Christian Bernard}

Nous disposons d'une vingtaine d'espaces monographiques dont les conditions d'assemblage, de présentation et d'évolution sont très variables. Certains espaces sont entièrement pris en charge par le musée (celui de Sherrie Levine, par exemple), d'autres doivent l'essentiel de leur forme et de leur fonctionnement à l'artiste. Le plus souvent, le travail est partagé. Il y a eu, en effet, le cas d'espaces "vides" dont la programmation était entièrement déléguée. L'un de ces cas est le Büro Kippenberger. Je souhaitais poursuivre avec Martin Kippenberger le travail commencé à la Villa Arson à Nice. Dès la fin 1990, nous avions arrêté le principe d'une exposition rétrospective (qui s'ouvrit quelques jours avant sa mort en 1996) et d'un espace permanent faisant mémoire du Büro Kippenberger de la fin des années 1970 à Berlin. Kippenberger décida donc de présenter dans sa salle de jeunes artistes, des figures de son entourage ou des artistes qu'il aimait. Il nous revenait simplement de mettre en œuvre cette programmation. Nous avons continué après sa disparition car sa liste n'était pas épuisée. Puis nous avons montré là des artistes que je savais appréciés de Kippenberger ou d'autres que j'espérais compatibles avec son esprit et son exigence. Il nous avait également déposé une partie importante de sa collection et donné l'ensemble des items constituant le MoMAS Projekte (le projet du Museum of Modern Art Syros). Toutes ces œuvres, souvent 
présentées, formaient la contribution variée de cet artiste au Mamco. L'espace monographique n'est qu'un aspect du travail d'un artiste dans le Mamco. Au-delà de son protocole spécifique, il implique d'autres formes de manifestation et de collaboration: publications, productions, expositions de formats divers, etc. L'espace monographique n'est pas un petit ghetto, c'est un "lieu en travail ", voué à déborder dans l'espace du musée.

De la même façon, la Suite genevoise est un ensemble de trois salles qui a été confié à l'ouverture du musée à John $M$. Armleder. Il y a proposé diverses expositions dont plusieurs l'ont associé à Sylvie Fleury et Olivier Mosset. Deux propositions d'Armleder pour la Suite genevoise sont aujourd'hui des souvenirs déterminants pour notre pratique de l'exposition du musée (Ne dites pas non! et Don't do it). Au bout de trois ans, la disponibilité d'Armleder pour cette activité s'est avérée difficile à entretenir. Nous avons interrompu ce mode de fonctionnement, mais pas la collaboration avec Armleder, Fleury ou Mosset. Outre de nombreux accrochages au fil du temps, nous avons réalisé deux grandes rétrospectives d'Armleder et de Fleury et nous en préparons une de Mosset. Cette logique d'alternance entre concentration et expansion vaut pour l'économie générale de l'occupation des salles à l'échelle du musée.

Le cas du Cabinet d'amateurs dédié à la Collection Yoon Ja et Paul Devautour, espace qui mimait, sous la forme d'un petit studio de deux pièces, une version "années quatre-vingt" de l'appartement de Ghislain Mollet-Viéville, est un peu différent. Durant plusieurs années, ce studio est resté très actif, toujours modifié et enrichi. Le travail de ce couple d'artistes était parfaitement en phase critique avec l'époque. La fin des années nonante les a vus se séparer et Paul Devautour a déplacé son travail vers le Net et d'autres pratiques comme la direction d'écoles d'art (aujourd'hui en Chine). Il n'y avait plus de sens à maintenir cet espace en l'état. Nous l'avons remisé il y a une dizaine d'années. L'ensemble des artistes fictifs dont Yoon Ja et Paul Devautour développaient le travail réel dressait un paysage possible de l'art de la fin du siècle dernier. C'était une sorte d'ironique pseudo-musée de 
l'air du temps. Le temps viendra bientôt où nous pourrons montrer à nouveau cette œuvre, mais ce sera en tant qu'objet historique, révolu, réinterrogeable depuis un moment très étranger à ses problématiques. J'attache beaucoup d'intérêt aux artistes déserteurs, ceux qui s'arrêtent, qui déboîtent, quittent la scène, bruyamment ou sans crier gare. Dans ce registre, nous avons aussi montré le travail du groupe Présence Panchounette qui s'est dissous en 1990 et dont nous espérons bientôt acquérir un large ensemble de pièces. Selon une autre procédure, nous avons pu implanter pendant deux ans le fameux ensemble des Thrift Store Paintings de Jim Shaw. Un autre simili ou anti musée au sein du Mamco.

L'agence Les Ready-made appartiennent à tout le monde ${ }^{\circledast}$ est un autre cas d'espace monographique permanent à composante évolutive. Je n'avais jamais travaillé avec Philippe Thomas auparavant, je ne l'avais même jamais rencontré. Pourtant, je suivais le développement de son œuvre avec intérêt et admiration. Je tiens par exemple l'exposition Feux Pâles au CAPC (Centre des arts plastiques contemporains) musée d'art contemporain de Bordeaux pour l'une des plus importantes expositions de cette époque. Nous allons d'ailleurs la reconstituer en 2011. C'est une des sources évidentes de Mamco, tant sur le plan théorique que sur le plan esthétique. Philippe Thomas était atteint du sida. Il avait décidé de clore à Lucerne l'activité de l'agence sous le nom de laquelle il opérait. Je suis allé lui proposer de refaire une ultime fois ce geste au Mamco et de réunir autant d'œuvres que possible dans ce dispositif à vocation permanente. Il est mort en 1995, quelques mois après l'ouverture du Mamco (1994). Pendant l'installation, il m'avait donné des indications pour que nous puissions continuer à faire "vivre" son espace. Et, de fait, nous n'avons cessé de le transformer en tâchant de rester fidèles à ses suggestions et à l'esprit de son travail. Après quelques années de "repos" et aussi de prêts à l'extérieur, nous allons réinstaller l'agence à la faveur de la rétrospective que nous préparons. Ce ne sera plus la même: l'époque a changé, nous avons changé, les objets n'ont plus la même contemporanéité. Son actualité dans le Mamco d'il y a presque 20 ans s'est transformée en historicité symptomatique. 


\section{Anne Bénichou et Francine Couture}

La question de la dernière œuvre est un thème récurrent dans le programme du Mamco. Pourquoi ?

\section{Christian Bernard}

Le musée ne saurait échapper à la question de la mort. Les tombeaux égyptiens, avec leurs "installations " de peintures murales et d'objets ne sont-ils pas de petits musées personnels? Les œuvres ne sont-elles pas des tentatives de passer outre la mort? Demander à un artiste par quel ensemble de ses œuvres il souhaiterait être représenté après sa disparition, c'est d'abord faire du musée un instrument pour l'artiste plutôt que l'inverse. C'est ensuite exprimer une attente qui excède les circonstances conjoncturelles. C'est enfin placer l'artiste devant la responsabilité de trier son œuvre d'un point de vue radical. La cruauté qui se joue là est une épreuve de vérité. J'ai posé la question de la " dernière salle " à plusieurs artistes, dont Parmiggiani, Rutault et Walther. Philippe Thomas y avait déjà répondu de facto. L'idée m'en était venue au Kunsthalle de Hambourg (avant son extension par Ungers). Walther, le grand artiste de la ville, y disposait d'une salle monographique d'une infinie tristesse. Cela m'a convaincu de l'importance qu'il y a à associer étroitement les artistes à la construction de leur mémoire. Cela ne signifie pas pour autant que le musée n'ait pas d'arguments critiques, scientifiques, historiques, esthétiques à faire valoir. Les réponses des artistes ont été très différentes les unes des autres. Parmiggiani a choisi de réaliser la Crypte, une œuvre qui, paradoxalement, représente un hapax dans son corpus. Rutault a sélectionné une de ses "définitions/méthodes" qui, de fait, les réunit toutes. Autrement dit, nous disposons, à l'intérieur du musée, de la totalité close de l'œuvre de Rutault. Avec Rutault, nous avons tout, charge à nous de déployer cette totalité; avec Parmiggiani, nous n'avons "rien", rien de ce qui emblématise son travail, même si la signification profonde de sa crypte éclaire singulièrement ce travail. La rétrospective Parmiggiani (1995) et les œuvres acquises dans son sillage nous ont permis d'en montrer tous les visages. 
Le cas du Werklager de Walther est aussi très éclairant. L'artiste a choisi de réunir dans sa salle un ensemble d'œuvres créées entre 1961 et 1972. Autrement dit, son ultima camera présente les dix "premières" années de son travail. Comme si ce qui précède et ce qui suit cette période y conduisait d'une part et s'en déduisait d'autre part, sans ajouter, au moins conceptuellement, à ce noyau stratégique. Il y a là une forme de lucidité exemplaire. Nous avons naturellement tenu à montrer dans des expositions complémentaires le travail en amont et en aval du Werklager, en révélant et publiant les Wortbilder de 1958-1959 et en dépliant toute l'œuvre, sur 33 salles et 1200 items, dans une rétrospective de 50 années de travail qui pouvait apparaître comme l'expansion à l'échelle du Mamco de la "dernière salle" initiale (2010). L'espace monographique de Walther présente d'autres aspects importants. Il contient une œuvre majeure de l'artiste, le Werksatz, qui y est montrée sous sa forme minimale de tas organisé en parallélépipède placé sous un capot de verre. Cette œuvre peut faire l'objet d'une présentation déployée au sol comme une vaste installation. Les éléments ainsi sortis de leurs sacs de protection sont alors disponibles pour un usage de performance. Trois états, trois modes d'existence d'une même œuvre dont on voit bien que le seul espace monographique ne saurait les contenir. C'est cette dialectique qui nous meut.

\section{Anne Bénichou et Francine Couture}

L'espace de l'atelier est également très présent au Mamco, en l'occurrence les espaces de Sarkis et de Cérard Collin-Thiébaut. L'atelier serait-il l'une des formes privilégiées de l'espace monographique?

\section{Christian Bernard}

Le type de l'atelier est évidemment emblématique des lieux pour l'art: lieu de production (le plus souvent), lieu de (première) monstration, lieu de débat, lieu de commerce, lieu de vie (parfois). C'est un mythème du monde de l'art, un théâtre où se scénographie l'acte de création. C'est ainsi que l'atelier est vite devenu un sujet pour la peinture. C'est un sujet pour notre musée. L'agence de Philippe Thomas présente un caractère d'atelier, autant dans sa partie 
"dépôt" que dans sa partie "showroom". Le Corridor Store Front de Christo (1966-1967) que nous présentons en permanence depuis 1994, ne l'oublions pas, a d'abord été installé dans l'atelier même de Christo. Il en était et métaphorisait la fonction marchande. Il faudrait également évoquer l'exposition d'Alain Bublex au Mamco. Elle comportait un atelier de menuiserie et de mécanique qui servait chaque semaine à produire des éléments nouveaux, insérés dans les salles. L'exposition comme atelier et vice versa.

Quant à l'espace monographique évolutif de Sarkis, nommé L'Atelier depuis 19380, il est en travail depuis l'ouverture du musée. Il n'a été fermé que durant trois mois sur 16 ans. En revanche, il a temporairement changé d'étage quand nous avons démonté les 20 salles en bois du premier étage pour faire place à une exposition de Fabrice Cygi (bientôt suivi par Vincent Lamouroux et Mike Nelson). En tant que salle "physique", cet atelier est aujourd'hui le dernier témoignage de la première structure matérielle du Mamco, l'époque héroïque (sic) des "cabanes". Il est donc un marqueur de notre fondation sous l'égide de l'expédient et de la pauvreté (comme Éros selon la Diotime de Socrate). Il suffit de visiter l'atelier de Sarkis à Villejuif pour se rendre compte qu'il est non seulement le creuset mais encore le lieu privilégié de son œuvre. Tout ici consone, s'articule, s'échange dans une étrange intimité polyphonique. Les expositions de Sarkis paraissent n'être que des extensions temporaires de ce qui est continûment à l'œuvre dans son atelier. C'est pourquoi je lui ai proposé de déplacer cet atelier au Mamco. Je me doutais que la réponse serait autre. Pourtant, c'est bien un atelier que Sarkis a créé au musée. Ce qu'il appelle un "atelier de voyage", une sorte d'excroissance extraterritoriale. Le titre exprime l'idée que Sarkis travaille en regard de la longue histoire des cultures humaines. Ses ressources, ses références excèdent depuis toujours la seule histoire de l'art. C'est pourquoi ses ateliers, ses expositions, ses œuvres ne cessent d'inclure des objets, des formes, des processus provenant de partout dans l'espace et dans le temps. Chaque année, Sarkis vient travailler dans son atelier deux ou trois fois. Il s'y enferme une journée durant. Il y réalise des aquarelles et des vidéos, il 
modifie la présentation de l'ensemble. Il suit un programme établi depuis longtemps et il l'infléchit au gré de ses circonstances. Nous avons un contrat moral avec lui. Nous rémunérons ses venues, nous achetons progressivement l'atelier et son contenu. Et comme pour les autres artistes disposant d'espaces monographiques, nous présentons des expositions concernant l'amont et l'aval de l'ensemble permanent. C'est ainsi que nous avons montré les œuvres «noires" des années 1969-1971 ou l'Atelier d'aquarelles dans l'eau et que nous allons déployer, ce printemps 2011, dans l'essentiel du musée, une large rétrospective de son œuvre prise sous l'angle de ce que l'artiste appelle ses "collaborations". J'aimerais encore ajouter qu'une amie de l'artiste a fleuri chaque semaine cet atelier durant 12 ans. Nous avons ensuite pris le relais. Faut-il dire que tous les changements opérés par Sarkis dans son espace font l'objet d'une documentation photographique aussi complète que possible? Aussi ce qui est voué à un mouvement permanent est-il comme suspendu au seuil de l'oubli par une archive iconographique.

\section{Anne Bénichou et Francine Couture}

Et l'atelier de Collin-Thiébaut, comment s'est-il développé?

\section{Christian Bernard}

Gérard Collin-Thiébaut vit près d'Ornans dans une maison assez grande pour lui offrir autant de pièces que de chantiers ouverts dans son travail. Un atelier par pratique: il passe de l'un à l'autre, de l'une à l'autre sans solution de continuité. Le projet de L'Atelier d'aujourd'hui était de rassembler dans un même espace (semi-permanent) une partie des pratiques quotidiennes de l'artiste, notamment la peinture (il réalise des puzzles des grands chefs-d'œuvre de l'histoire de l'art) et l'écriture (il recopie à la main sur des cahiers d'écolier les 17000 pages du journal d'Amiel). Vous noterez que la Collection Yoon Ja et Paul Devautour, Présence Panchounette, Collin-Thiébaut (et même, dans une autre mesure, Philippe Thomas) forment une constellation qui dessine un contrepoint critique (et français) aux appropriationnistes étatsuniens que par ailleurs le Mamco montre amplement. L'Atelier d'aujourd'hui, qui jouxte celui de Sarkis, doit son titre au clin d'œil " parricide" de Collin-Thiébaut à 
ce dernier qui fut son mentor décisif. Cet atelier se présente comme une grande chambre bourgeoise: portes, plafond, plafonnier, plinthes, mobilier "cosy", tapis, porte-manteaux, bureau, etc. Rien de la nudité qui caractérise les autres espaces du musée, sinon l'appartement de Ghislain Mollet-Viéville. Au fil du temps et des réaccrochages, façon salon de peinture du XIX ${ }^{e}$ siècle, des œuvres réalisées sur place, les murs ont été insensiblement assombris, accélérant symboliquement l'œuvre du temps. Nous avons également un contrat avec l'artiste qui nous permet de défrayer (légèrement) le travail qu'il réalise pour son atelier genevois. Par ailleurs, à côté de sa présence récurrente dans nos autres accrochages, nous préparons la publication d'un livre étonnant, où il recense et décrit l'ensemble des ses travaux.

\section{Anne Bénichou et Francine Couture}

Les modifications que vous évoquez ont-elles été faites à la suggestion de l'artiste?

\section{Christian Bernard}

Oui, pour certaines. Pour d'autres, il s'agit de propositions que nous lui faisons. Il a pu arriver que tel changement ponctuel soit de notre seul fait: c'est une collaboration qui repose sur un très long compagnonnage (je connais CollinThiébaut depuis 1968). Pour ne prendre qu'un exemple, c'est nous qui lui avons suggéré d'accueillir dans son atelier des œuvres "voisines". C'est ainsi que l'on a pu y voir des pièces d'Ernest T., de Dimitrijevic, d'André Raffay ou de Sherrie Levine, par exemple. L'esthétique "cabinet d'amateur" de l'univers de Collin-Thiébaut a naturellement absorbé ces invités.

\section{Anne Bénichou et Francine Couture \\ Est-ce Collin-Thiébaut qui a invité les artistes?}

\section{Christian Bernard}

Je lui ai proposé des pièces, il en a parfois préféré d'autres. Le problème des espaces monographiques permanents ou récurrents, c'est évidemment l'usure que cette durée provoque inévitablement. Usure des regardeurs comme des artistes. L'expérience montre que les artistes (Sarkis mis à part) 
n'ont pas indéfiniment le désir de réinventer leur espace. Ils ont d'autres sollicitations, l'œuvre suivante est souvent bien plus excitante. C'est une contradiction insurmontable. La solution que nous avons adoptée consiste alors à remiser temporairement ces unités monographiques en attendant que leur absence suscite l'attente de leur retour. Je crois que toutes les œuvres soumises durablement au regard tendent à s'effacer de celui-ci. Que regardons-nous des tableaux qui sont sur nos murs domestiques et que nous voyons tous les jours? Quand nous réimplanterons L' Atelier d'aujourd'hui, ce devra être comme une nouvelle première fois.

\section{Anne Bénichou et Francine Couture \\ Vous voulez éviter la fétichisation ou la fixation?}

\section{Christian Bernard}

Je souhaite surtout que les visiteurs aient envie de revenir, de regarder à nouveaux frais. Rien n'est pire que de voir un visiteur passer la tête par la porte et dire "Ah, j'ai déjà vu ça." Le sentiment de déjà-vu, au musée, c'est intéressant quand on le recherche, mais c'est affligeant quand il trahit une satiété, une inappétence ou une lassitude. D'une façon générale, j'essaie de travailler contre les mécanismes de fétichisation et contre le sentiment de fixité qui y contribue. Rien de ce qui emphatise ou sacralise les œuvres ne me paraît compatible avec l'idée que je me fais d'un musée profane et démocratique.

\section{Anne Bénichou et Francine Couture}

Ces œuvres «invitées " font-elle partie de la collection du Mamco?

\section{Christian Bernard}

De la collection, qu'il s'agisse d'acquisitions ou de dépôts à long terme, sauf pour une pièce que j'ai empruntée à l'État français, La Bohémienne endormie de Henri Rousseau par André Raffray, œuvre vraiment magnifique.

\section{Anne Bénichou et Francine Couture}

Ce serait également une façon de réactiver les pièces de la collection, de les relire constamment? 


\section{Christian Bernard}

Exactement. Toutes les œuvres invitées chez Collin-Thiébaut avaient été présentées auparavant selon des configurations différentes. Par exemple les deux After Mondrian de Sherrie Levine et ceux d'Ernest T. avaient été exposés précédemment dans une salle très "déceptive" qui réunissait des œuvres "à la manière de Mondrian". Il s'agissait plus de démonter l'idée d'appropriation que de l'illustrer sur un mode valorisant. Les "Douanier Rousseau" d'Ernest T. ont d'abord fait l'objet d'une exposition exhaustive. Dans l'atelier, on n'en a montré que quelques-uns. Les différents contextes changent ou étoffent les significations des œuvres, c'est bien connu. Un des axes de notre travail expositionnel consiste à multiplier les contextualisations. Cela ne peut s'opérer sans changement de registre herméneutique ni sans risques.

\section{Anne Bénichou et Francine Couture}

Ã travers ces scénographies, cherchez-vous à provoquer une fusion entre l'œuvre autonome et le cadre d'exposition ? On arrive souvent mal à départager les deux.

\section{Christian Bernard}

Je ne crois pas à l'œuvre autonome. Une pièce unique dans un white cube ou une fresque de Fra Angelico dans une cellule de San Marco ne sont pas autonomes : elles sont tressées dans une conception du monde et de l'art qui détermine leur régime de visibilité. Un mur blanc est un contexte aussi puissant qu'un mur saturé d'œuvres diverses. Tout dépend de l'intériorisation des normes d'expression et d'exposition. Le travail expositionnel muséologique a pour objet d'expérimenter les interrelations potentielles entre les œuvres. D'une manière ou d'une autre, les œuvres sont toujours exposées. C'est la condition de leur existence. Nous réglons les intervalles entre les œuvres, nous prédisposons des rushes dont le regardeur va réaliser le montage. Personne ne voit jamais la même exposition. À chacun son film.

\section{Anne Bénichou et Francine Couture}

Sans qu'il y ait de démarcation, il n'y a pas de séparation entre le contenant et le contenu. 


\section{Christian Bernard}

Le musée est un médium et l'on sait que c'est donc aussi un message. Chaque donnée visuelle de l'espace muséographique relève de l'énonciation artistique. Cela ne veut pas dire que le scénario expositionnel soit une œuvre, cela signifie que rien de l'espace pragmatique du musée n'est exclu des mécanismes de mise à vue des œuvres.

\section{Anne Bénichou et Francine Couture}

Vous avez également reconstitué au Mamco l'appartement-galerie que Chislain Mollet-Viéville avait occupé au 26, rue Beaubourg à Paris, de 1975 à 1991, dans lequel il vivait et exerçait ses activités de galeriste d'art conceptuel. Qu'est-ce qui a motivé cette reconstitution?

\section{Christian Bernard}

Ce projet s'inscrit dans une histoire qui précède celle du musée. Cet appartement est passionnant pour de nombreuses raisons. D'abord, parce que, du point de vue d'une politique esthétique générale, nous pouvons constater, dans la vieille Europe, que les gens les plus orientés vers l'art moderne et contemporain continuent de vivre dans des environnements très imprégnés des formes et des objets du passé. Il suffit de voir les photographies représentant les grandes collections privées. Bien que les choses aient évolué, il est rare de trouver chez un collectionneur ou un acteur de l'art un accord continu entre l'ensemble des formes de la vie matérielle et des choix esthétiques et artistiques. C'est plus facile d'en trouver chez les artistes ou les critiques. Il y a évidemment des exemples remarquables, mais ils restent peu nombreux. Donc, je trouvais très important de montrer ce que pouvait être l'intérieur de quelqu'un qui mettait l'ensemble de ses décisions au diapason de ses choix artistiques.

De plus, cet appartement était devenu un objet historiquement clos. Ghislain Mollet-Viéville y avait renoncé pour engager une nouvelle étape de sa vie qui tirait davantage encore les conséquences de ses orientations fondamentales. Si des œuvres dont il se fait le thuriféraire et le spécialiste se tiennent déjà tout entières dans leur certificat, autant 
s'en tenir au certificat et ne plus avoir d'œuvres chez soi. Et du coup, il a déménagé dans un nouvel appartement (peutêtre aussi pour d'autres raisons). Dans ce nouvel espace, il n'y a plus rien à voir mis à part Mollet-Viéville, ses armoires, son bureau, mais certainement pas d'œuvres au mur, ou alors de façon purement transitoire et indicielle.

Pour le micro-milieu d'avant-garde européen et français des années septante, l'appartement situé en face du Centre Pompidou est devenu un lieu de mémoire. Il a disparu tout en persistant dans l'esprit des artistes et des amateurs qui s'y sont informés ou formés. Par ailleurs, je connaissais bien Mollet-Viéville parce qu'il était attentif au travail que je conduisais à la Villa Arson. Dans ce cadre-là, il m'a souvent accompagné dans les voyages que j'organisais en Europe ou aux Etats-Unis pour visiter des expositions et des institutions. Nous avions un lien amical qui a évidemment facilité les choses. Son appartement était un lieu exemplaire qui avait disparu et qui méritait d'être remémoré dans son intégralité. Il y avait à la fois un contenant extrêmement adéquat et un contenu très important pour le musée qui ne disposait pas d'œuvres de ce type quand il a ouvert ses portes.

\section{Anne Bénichou et Francine Couture}

L'appartement de Chislain Mollet-Viéville était également un espace de vie. Cherchez-vous à préserver quelque chose de cette valeur d'usage?

\section{Christian Bernard}

Je voulais de surcroît utiliser la dimension fonctionnelle de l'appartement, faire glisser les frontières entre ce qui est simplement à considérer et ce dont on peut faire usage sans suspendre la considération (non sidérée). Comme cet appartement avait été le théâtre de nombreuses rencontres, discussions, événements, il fallait poursuivre dans cette voie. Ainsi, nous y organisons des lectures, des rencontres, des concerts, des discussions et autres conférences de presse. Il fallait que ce soit aussi un lieu d'usage banalisé, où des gens se donnent librement rendez-vous ou viennent tout simplement lire. Cela fonctionne bien 
maintenant. J'aurais aimé que cet usage se diffuse plus spontanément. On ne peut pas dire au gens : "Asseyezvous" ou "Venez lire ici " ou adresser des injonctions de ce type. On mesure d'ailleurs là l'autorité "distanciante" que représente cette esthétique "minimaliste" par rapport au visiteur. Elle crée une forme de séparation. Bien sûr le contexte du musée inhibe déjà. Certains enseignants ont pris l'habitude de l'utiliser avec leurs élèves; il se passe désormais beaucoup de choses dans L'Appartement, bien audelà de la simple visite.

\section{Anne Bénichou et Francine Couture}

Comment avez-vous procédé pour mener cette reconstitution? Quelles modifications y avez-vous apportées depuis son ouverture?

\section{Christian Bernard}

Ce qui nous intéressait était évidemment de le reconstituer, mais pas littéralement. Nous en avons augmenté la taille parce qu'il était évidemment plus petit à Paris. Et la mémoire grandit les choses que la vie a aimées. C'est aussi pour cela que nous l'avons fait plus spacieux. Ma préoccupation était de pouvoir en faire divers usages. Je n'avais pas à l'époque l'inquiétude de son évolution. Très tôt, Mollet-Viéville a évoqué l'idée que nous puissions le modifier régulièrement, $\mathrm{y}$ insérer des choses nouvelles selon les occasions, les suggestions et l'imagination. On ne peut pas dire que sa délégation d'aménagement soit totale. MolletViéville exerce un contrôle amical sur l'évolution de l'appartement, même si je force un peu sa main de temps en temps. Je reste toujours dans le cadre de ce qu'il est susceptible d'accepter volontiers. Dans certains cas, il a été plus réticent que dans d'autres. Mais en même temps, ce n'est plus chez lui, même si c'est toujours à lui.

Nous avons un peu modifié la structure de l'appartement afin de lui donner plus de simplicité. Certaines œuvres ont été déplacées pour des raisons de conservation préventive, notamment le Don Judd qui était vraiment problématique puisque tout le monde le touchait. Cela pouvait l'abîmer. On a mis à l'abri les œuvres les plus fragiles. J'ai également 
traité le bureau d'une façon très particulière, sans aucun mimétisme de ce que pouvait faire Mollet-Viéville, mais sans remettre en question la fonction de ce bureau où il y avait beaucoup de choses entassées au fil des circonstances. J'ai pris comme modèle L'Enseigne de Cersaint dont nous avons emprunté la gravure au Cabinet des estampes. Nous avons donc réalisé un accrochage all over en hommage à Watteau et aux plus ou moins petits commerçants de l'art dont Mollet-Viéville est une version contemporaine.

\section{Anne Bénichou et Francine Couture}

Donc, vous parlez de lui, vous le mettez en scène. Avezvous consulté les artistes ou seulement Chislain MolletViéville?

\section{Christian Bernard}

Au départ, uniquement Mollet-Viéville. Il est arrivé que l'on installe telle ou telle pièce d'un artiste plus jeune, avec sa participation directe ou indirecte, mais L'Appartement n'est pas un lieu de coopération courante avec les artistes comme le sont les autres espaces du musée. C'est le lieu du collectionneur et de sa collection; c'est pourquoi on y insère des choses compatibles, non coruscantes. Luimême nous a parfois suggéré des artistes. Nous avons parfois donné suite, mais pas toujours. Il est plus indulgent que nous pour des artistes qui sont aujourd'hui dans une situation tardive et épigonale. Je comprends bien qu'il y voie une continuation de ses choix de jeunesse. Nous avons introduit peu d'éléments hétérogènes. Ces insertions sont parfois durables, parfois juste ponctuelles. L'idée est de garder l'esprit de l'appartement le plus intègre possible, de ne pas y glisser trop d'embarras, de perturbations. Il y a assez de lieux au Mamco où l'on éprouve ce type de larsen. Il faut que le visiteur perçoive l'unité d'ensemble de l'appartement. Au fil du temps, il s'est transformé, mais lentement, insensiblement. Il a connu plus de 36 états successifs et pourtant c'est toujours le même. Ainsi du Mamco qui change au trois-quarts trois fois par an et qui demeure pourtant semblable dans le dédale toujours recommencé de son réseau d'expositions communiquant entre elles. 